\title{
Design and optimization of a semi-active suspension system for railway applications
}

\author{
Benedetto ALlOTtA ${ }^{1}$, Luca PUGI ${ }^{*}$, Valentina COLLA ${ }^{2}$, Fabio BARTOLINI $^{1}$, Francesco CANGIOLI ${ }^{1}$ \\ 1. Department of Energy Engineering “S. Stecco”, University of Florence, Florence, Italy \\ 2. Perceptual Robotics Laboratory, Sant'Anna School of Advanced Studies, Pisa, Italy
}

\begin{abstract}
The present work focused on the application of innovative damping technologies in order to improve railway vehicle performances in terms of dynamic stability and comfort. As a benchmark case-study, the secondary suspension stage was selected and different control techniques were investigated, such as skyhook, dynamic compensation, and sliding mode control. The final aim was to investigate which control schemes are suitable for optimal exploitation of the non-linear behavior of the actuators. The performance improvement achieved by adoption of the semi-active dampers on a standard high-speed train was evaluated in terms of passenger comfort. Different control strategies have been investigated by comparing a simple SISO (single input single output) regulator based on the skyhook damper approach with a centralized regulator. The centralized regulator allows for the estimation of a near optimal set of control forces that minimize car-body accelerations with respect to constraints imposed by limited performance of semi-active actuators. Simulation results show that best results is obtained using a mixed approach that considers the simultaneous applications of model based and feedback compensation control terms.
\end{abstract}

Key words: Magneto Rheological dampers, gradient projection method; semi active suspension systems; skyhook

C 2011 JMT. All rights reserved.

\section{Introduction}

$\mathrm{L}$ ooking at the automotive commercial products and at the last innovative railway applications, Magneto-Rheological (MR) damper is a promising technology [1-2], which has several advantages with respect to the classical passive or active suspension system such as:

- There are no mobile parts, and thus the actuator is quite simple, small and robust.

- It is a controlled damper; therefore, the power needed to obtain the required force is quite small.

- Being a damper, it can only dissipate energy and cannot inject energy in the mechanical system, which makes the control system less sensitive to "chatter" and "spill over" problems.

- In case of fault, a "fail safe" behavior can be easily obtained, as the suspension system can continue to work in a passive way with degraded performances.

On the other hand, this kind of suspension system also has some limitations with respect to an active actuator:

\footnotetext{
Received Aug. 31, 2011; revision accepted Oct. 28, 2011

${ }^{*}$ Corresponding author. Tel.: +39-3482106954

E-mail: pugiluca@libero.it (L. PUGI)

(C) 2011 JMT. All rights reserved

doi: 10.3969/j.issn.2095-087X.2011.04.002
}

- The force exerted by the MR damper is mainly a function of the control current, the damper velocity and the fluid temperature; thus non-linear behavior may arise during the control phase of the system.

- The output force is dissipative and can be exerted only when force and velocity have opposite directions (resulting power is negative corresponding to a dissipation effect).

- In order to ensure a mean or static operation point, this kind of actuator needs to work with a spring or another force element.

Despite these limitations, MR dampers are a good solution for semi-active suspension systems in automotive applications [3-5]. They are also applied in new implementations and revamp of existing vehicles, and they are also applied for military vehicles [6]. The purpose of this article is to investigate the feasibility and the possible improvements deriving from the application of semiactive suspension systems with MR damper to high speed railway vehicles. A generic multi-body train model was developed in Matlab-SimMechanics ${ }^{\mathrm{TM}}$ to simulate and verify performances of different control systems. The model was derived from previous research activities [7-8]. Semi-active dampers are supposed to be applied in parallel to the secondary stage of the vertical suspension system which is modeled as an equivalent 
passive spring-damper element. Three different control strategies are discussed:

- Skyhook [9]: each damper works independently in maintaining a steady position of the controlled body.

- Dynamic compensation: the dynamic behavior of the vehicle car body is estimated using a simplified linear model. In this model, a simple plate is connected to a movable ground by means of spring and MR dampers that are actuated in order to minimize the dynamic behavior of the system.

- Dynamic compensation with acceleration feedback: the dynamic compensation is coupled with a PID controller in order to reject the contribution of non-linear components. These components include bump-stops, which cannot be simulated by the online model used in dynamic compensation strategy.

The proposed control strategies are compared by evaluating the performance of the control on a straight track with a length of about $700 \mathrm{~m}$ at different travelling speeds and three-dimensional irregularities. In addition, curved track tests are performed in order to verify that the proposed solution works on different line designs. The control is to be applied to the secondary vertical suspension stage which mainly affects vehicle comfort. As a consequence the work is more focused on comfort evaluation, since stability is not directly affected by the proposed solution.

This paper is organized as follows: Section 2 summarizes the vehicle and actuator models that have been used throughout the work. Section 3 describes the conditions under which the different control strategies have been tested and the index that have been adopted in order to quantify and compare the control performances. Section 4 is devoted to a theoretical description of the different control approaches that have been applied. Section 5 compares the performance of the applied control strategies by discussing some numerical results. Finally Section 6 presents some concluding remarks.

\section{Modeling}

The simulations are performed with models developed in Matlab-Simulink ${ }^{\mathrm{TM}}$ and in particular the SimMechanic Blockset ${ }^{\mathrm{TM}}$. Since the chosen development tool is not specifically developed for railway applications, some additional blocks have been introduced to simulate the behavior of many non-linear components/ phenomena, such as wheel-rail contact, non-linear damper, spring elements and finally, the MR dampers.

\subsection{Vehicle modeling}

The vehicle is depicted by a typical layout (see Fig. 1): the car-body is sustained by two bogies, each with

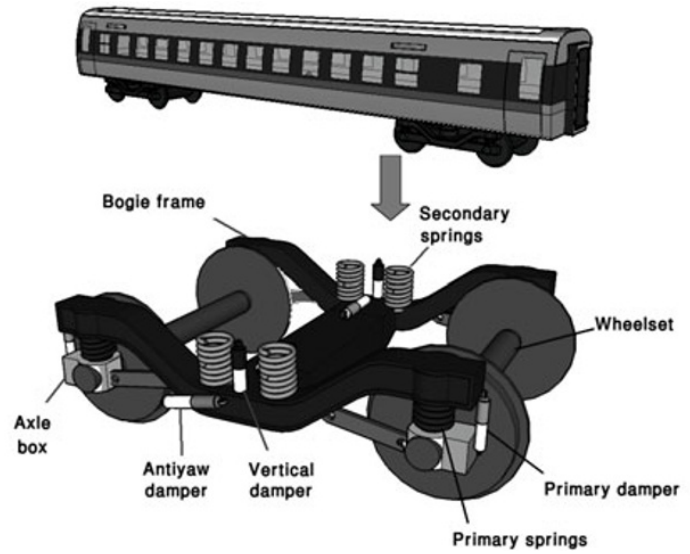

Fig. 1 Train vehicle and bogie layout

two axles. Two stage suspension systems are utilized for the vehicle with lateral and anti-yaw dampers. Roll bars are used to prevent excessive rolling motions. Primary suspension stage is placed on the axle box with two oscillating arms attached to the bogie frame by means of rubber elements (which is named sutuco) and is composed of a damper and a shear spring. This is vertically oriented and is attached to the upper part of the axle-box. The secondary suspension stage is realized by means of a pair of springs acting between the frame and a bolster which is attached to the carbody.

A Watt's linkage for the transmission of longitudinal efforts between the carbody and the bogie frame is connected to the lower part of the bolster. The damping ratio is guaranteed by vertical, lateral, and anti yaw dampers. In order to avoid the rotation and excessive displacement of the car body, an antiroll bar and bump stops have been applied to both vertical and lateral suspensions. The wheel rail contact is realized using a standard ISO ORE S1002 wheel profile and an UIC60 with cant $1 / 20$ rail geometry. The block structure of the model developed in Simulink is useful to have a flexible and easy customizable multi-body model. This is a considerable simplification of the control system implementation and calibration. For a satisfactory implementation of most of the model components, the classic library elements have been modified. The main data of the vehicle concerning geometry, inertial properties, damping and stiffness of suspension elements are taken from realistic applications and are available in previous publications of the authors [8]. The linkages between the bodies of the model are realized through prismatic and revolute links, in order to reproduce the kinematic behavior of the bodies. The wheel rail contact force is calculated by means of an algorithm that was developed in [7] according to Kalker theory [10]. The force elements, which are modeled to reproduce two suspension stages, have been introduced in the form of customized spring-damper blocks, and are capable of reproducing the typical nonlinear characteristics of the real elements. The active dampers are modeled in order to reproduce a damping 
factor that varies according to the current command. Moreover, bump-stops are introduced, such as a nonlinear force spring with a non-force gap near the noninterference position. In addition, accelerometers and inclinometer sensors are modeled considering a placement both on bogies and carbody, in order to test the train behavior and realize the input for the control system.

\subsection{MR-damper model}

MR dampers are realized in order to exploit properties of magneto-rheological fluids [2]. Viscous properties of these fluids are affected by the magnetic field applied to them, at least until the saturation of the ferromagnetic fluid particles arise. One of the first direct applications of these fluids was in the realization of a damper with adjustable damping coefficient. In this device, by changing the value of the magnetic field of the narrow conduct of the damper, the value of the damper force can be dynamically controlled by a semi-active actuator without any movable mechanical part. In order to implement the MR dampers inside the Simulink model of the train, the equation coefficients have been previously tuned by means of finite element methods through a multi-physics program (such as $\mathrm{Comsol}^{\mathrm{TM}}$ ). This tuning procedure has led to an implementation of the MR fluid behavior in the form of a Bingham liquid [11], where the relationship between the fluid stress and $\tau$ the magnetic field $H$ can be evaluated according to (1):

$$
\tau=\tau_{I}(H)+\eta \dot{\gamma}
$$

where $\tau_{I}(H)$ is a term that depends on the value of the magnetic field $H$ in the fluid, $\eta$ is the viscosity of the fluid when no magnetic field is applied, and $\dot{\gamma}$ is the fluid shear rate. The Bingham's model (1) is valid under the assumption of fluid incompressibility. Consequently, the elastic-compressibility phenomenon with low shear rate fluid stress is evaluated as $\tau=G \gamma$, where $G$ is the tangential-elasticity modulus of the fluid. The values of $\tau_{I}(H)$ for MR dampers can be assumed roughly proportional to the field $H$, until the saturation of the ferromagnetic fluid particles is reached. Therefore, from the control point of view, the value of the pressure drop/losses of the fluid flow inside the dampers can be easily controlled by regulating the value of the current $I$ through a simple solenoid. The force exerted by this kind of damper, due to the high value of $\tau_{I}(H)$, is quite insensitive to disturbances of deformation $\dot{\gamma}$ and temperature $T$. In particular, Eq. (2), which considers the temperature disturbance negligible, is assumed as follows:

$$
F=F_{0} I \operatorname{sign}(\dot{\gamma})-\eta \dot{\gamma}
$$

The viscous damping coefficient $\eta$ can be small or negligible when compared to the controlled term $F_{0} I$, so the controlled force $F$ can be easily considered roughly proportional to the control current $I$, as in the case of a perfect force-controlled actuator until the behavior of the damper is dissipative. Of course, the dynamic behavior of the fluid and the electric circuit has to be taken into account. From the literature results, MR fluid components delay can be easily modeled with a first order filter as visible in (3):

$$
I=\frac{I_{\mathrm{ref}}(s)}{1+t_{\mathrm{ref}} s},
$$

where $s$ is the Laplace operator and $I_{\text {ref }}(s)$ is assumed to be the reference current command corresponding to the desired force exerted by the actuator. Dynamic behavior of the filter (3) is tuned by setting the time constant $t_{\mathrm{d}}$ to a value of about $10-20 \mathrm{~ms}$.

\section{Testing conditions and performance indices}

In order to test the vehicle performance when the different control strategies are applied, a $700 \mathrm{~m}$ straight railway track with irregularities has been simulated. Three-dimensional irregularities are obtained by superimposing different kinds of disturbances, whose profiles are extracted from previous studies and inspired by realistic operating conditions [8].

The following types of irregularities can be distinguished:

- Vertical irregularities: both rail lines of the track have vertical displacements.

- Twist irregularities: the track plane is rotated with respect to an axes oriented along the track.

- Lateral irregularities: both rail lines have lateral displacements with respect to the original track.

- Gauge irregularities: only one of the rail lines is moved perpendicularly to the railway track.

Amplitude and spectral content associated with the irregularities are deliberately chosen to affect the nonlinear behavior of the suspension system (a low frequency, high amplitude displacement is deliberately inserted to activate carbody bump-stops). A reference irregularity path $700 \mathrm{~m}$ long has been defined with a sampling of $0.1 \mathrm{~m}$ as shown in Fig. 2. The chosen irregularity path is a compromise between realistic results and optimum computational effort, which is needed for iterative calibration and testing procedures for different control algorithms. 


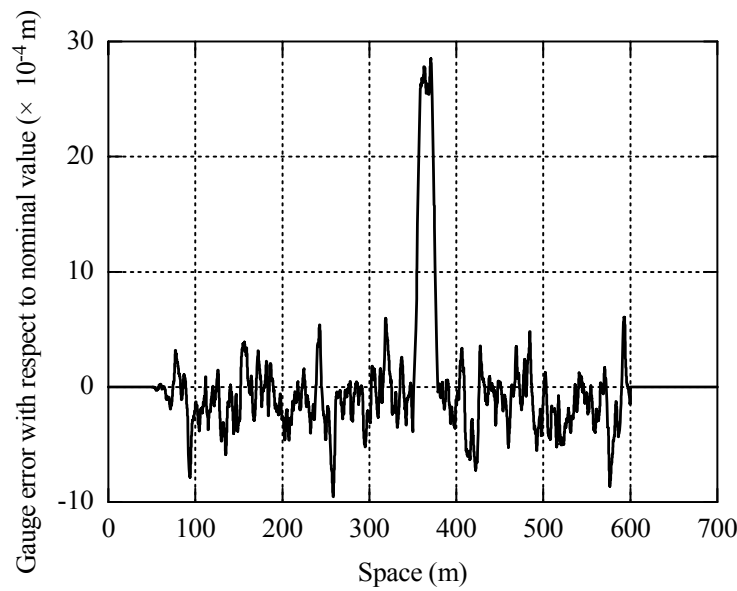

(a) gauge irregularities

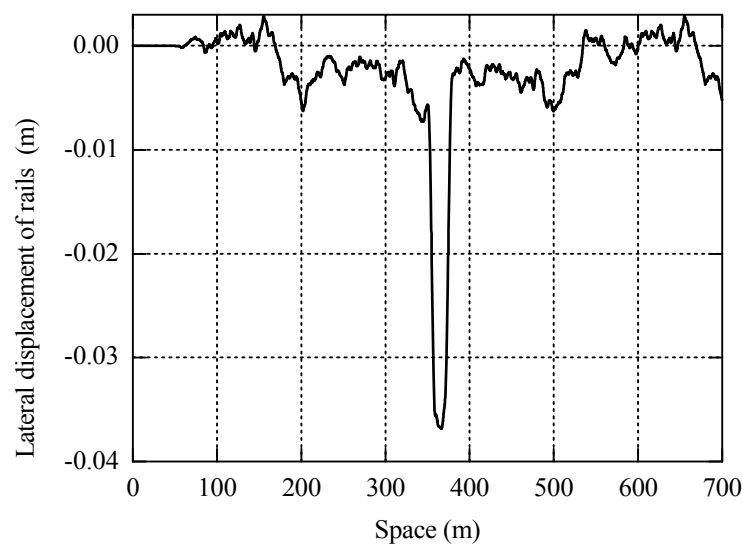

(c) lateral irregularities

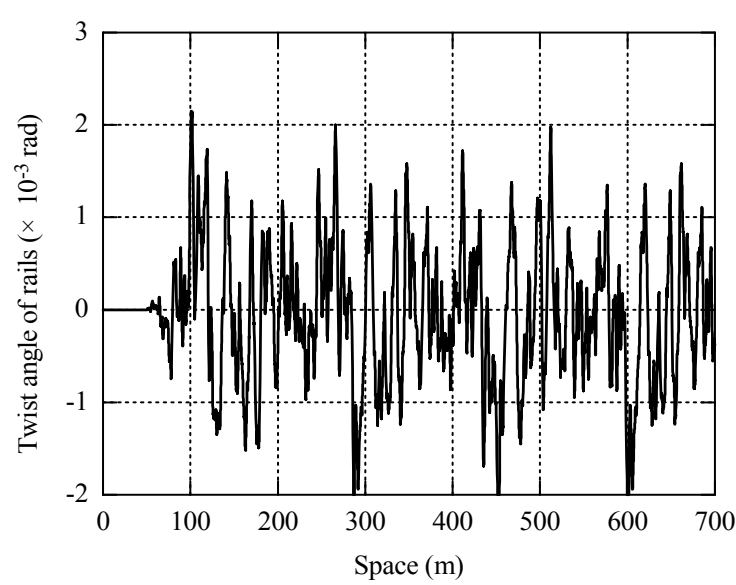

(b) twist irregularities

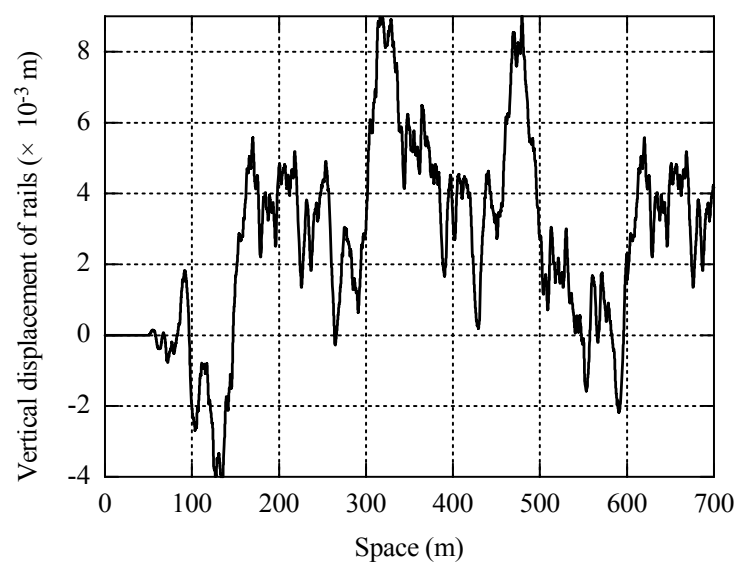

(d) vertical irregularities

Fig. 2 Reference irregularities paths

In order to evaluate and calibrate the proposed control strategies, the simulation results were processed to obtain some performance indices derived by regulation in forces [12]. This kind of analysis allows the evaluation of an imposed acceleration index experienced by railway passengers along their travel. For this evaluation, the acceleration values must be measured on the three reference axes of the vehicle, in three different points placed as depicted in Fig. 3 on the rear, central, and frontal parts of the carbody. A sensor layout is chosen, which is recommended by widely accepted international standards [12].

Then, taking into account the different perceptions of the human body to the acceleration at different frequencies, the value of the measured acceleration is filtered. The applied filtering functions, the bode diagrams of which are reproduced in Fig. 4, are suggested by widely

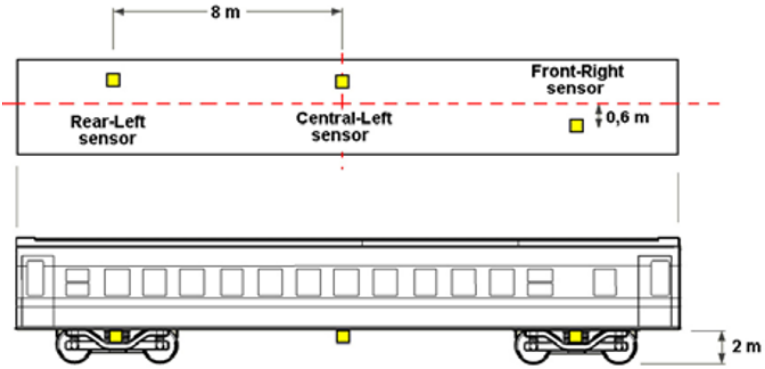

Fig. 3 Layout of acceleration sensors on carbody for evaluating comfort performances

diffused and accepted comfort standards [12].

In order to fully understand the vehicle behavior, performance indices defined according to (4) are introduced: 


$$
\left.\begin{array}{l}
I_{1}=\sqrt{r m s\left(a_{1 x}\right)^{2}+r m s\left(a_{1 y}\right)^{2}+r m s\left(a_{1 z}\right)^{2}}, \\
I_{2}=\sqrt{r m s\left(a_{2 x}\right)^{2}+r m s\left(a_{2 y}\right)^{2}+r m s\left(a_{2 z}\right)^{2}}, \\
I_{3}=\sqrt{r m s\left(a_{3 x}\right)^{2}+r m s\left(a_{3 y}\right)^{2}+r m s\left(a_{3 z}\right)^{2}}, \\
I_{x}=\sqrt{r m s\left(a_{1 x}\right)^{2}+r m s\left(a_{2 x}\right)^{2}+r m s\left(a_{3 x}\right)^{2}}, \\
I_{y}=\sqrt{r m s\left(a_{1 y}\right)^{2}+r m s\left(a_{2 y}\right)^{2}+r m s\left(a_{3 y}\right)^{2}}, \\
I_{z}=\sqrt{r m s\left(a_{1 z}\right)^{2}+r m s\left(a_{2 z}\right)^{2}+r m s\left(a_{3 z}\right)^{2}},
\end{array}\right\}
$$

where accelerations $a_{i j}$ are defined in the following way: the sub-index $i$ represents sensor placement (1-3), and the sub-index $j$ the corresponding measured scalar component.

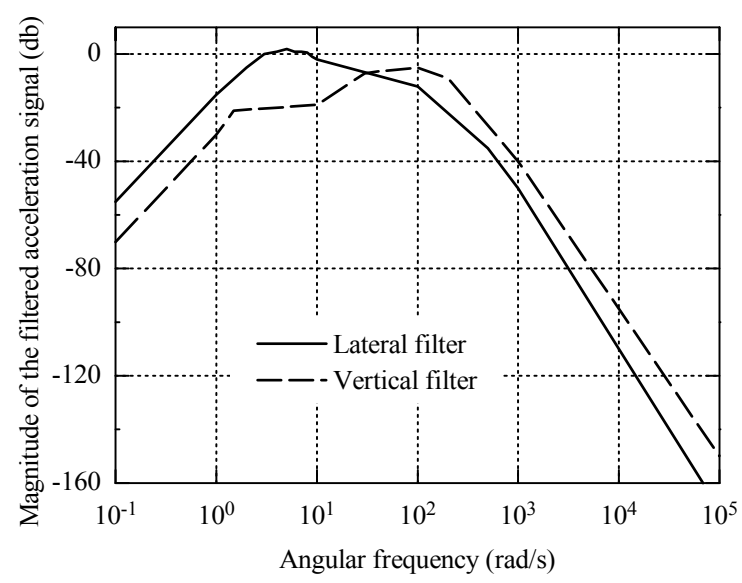

Fig. 4 Bode diagrams of lateral and vertical accelerations

\section{Proposed control strategies}

In order to reduce the accelerations experienced by passengers on a train carbody running on a railway with irregularities, it is proposed to use four vertical MR dampers placed in parallel to secondary suspension springs. Three different kinds of control strategies are proposed and compared in this work: skyhook, dynamic compensation, and sliding mode. In particular, the proposed skyhook regulator, which is described in Section 4.1 , is "local," in the sense that each MR damper works independently of the others. On the other hand, the other two strategies, the dynamic compensation and the sliding mode discussed in Sections 4.3 and 4.4, are "centralized" since they actually exploit the same velocity measurements of skyhook control, but such variables are the input of a MIMO (multiple-input and multiple-output) regulator which outputs an optimal set of damping forces that should minimize the acceleration of the whole carbody. To this end, a real-time simplified model of the controlled system that calculates the dynamics that have to be compensated by the regulator is needed. On-line estimator of plant dynamics is described in Section 4.2.

\subsection{Skyhook}

This kind of algorithm [9], which is widely adopted for suspension control problems, works with an SISO (single input single output) logic. Therefore, each active vertical damper works independently of the others, by exerting a force $F$ that is directly proportional to the absolute velocity of the carbody measured on the corresponding damper link $\dot{x}_{c}$ :

$$
F=-c_{x} \dot{x}_{c}
$$

Since an MR damper can only dissipate power, the force is applied only if the product of force and relative velocity $v$ is negative:

$$
P=F v=-c_{x} \dot{x}_{c} v<0 \leftrightarrow \dot{x}_{c} v>0 .
$$

The application of Eq. (6) involves measurement of the speed of both ends of the actuator (the former one linked to the carbody $\dot{x}_{c}$ and the latter $\dot{x}_{b}$ to the bogie).

Let us suppose that the corresponding accelerations are measured through inertial sensors and the velocities are extracted through integration as follows:

$$
\begin{aligned}
& \dot{x}_{c}=\int \ddot{x}_{c} \mathrm{~d} t, \\
& v=\int\left(\ddot{x}_{c}-\ddot{x}_{b}\right) \mathrm{d} t .
\end{aligned}
$$

The implementation of the proposed Skyhook control is quite simple in terms of computational resources, calibration complexity, and required hardware. Also the calibration and optimization phases are relatively simple with this kind of logic, since only the skyhook constant $c_{s}$ has to be chosen. Finally, this kind of control strategy ensures a fail-safe behavior because of its redundant structure: the MR damper, even in case of failure, keeps its natural residual damping function, and thus the system can continue to work with acceptable performance when three dampers are in controlled mode and the left one works in passive mode.

\subsection{On line estimator of plant dynamics}

A simplified scheme of the model assumed by plant estimator is shown in Fig. 5: the carbody is assumed to be a rigid body with three degrees of freedom; $2 a$ and $2 b$ are the corresponding distances between the four actuators; $m$ is its mass; and, $I_{x x}$ and $I_{y y}$ are the moments of inertia with respect to the first two axes of the reference frame. MR actuators are modeled as identical monodimensional elements. The quantities $z_{i}$ (where $i=1,2,3,4$ ) are the coordinates of each actuator along the $z$ axis, and the values $w_{i}$ (where $i=1,2,3,4$ ) are the associated external disturbances. 


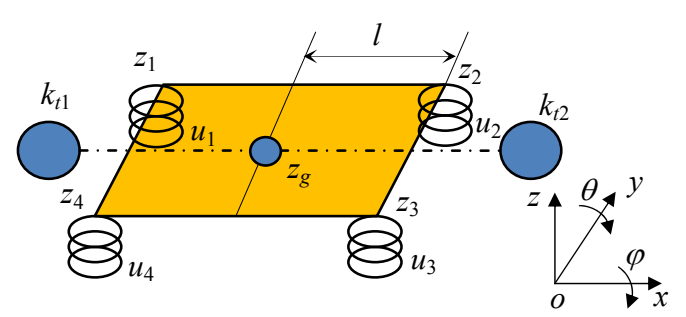

Fig. 5 Simplified model used by the estimator

Actuators' end positions $z_{i}$ are coupled by the geometrical relations (9) with the vertical components of the position of mass center $z_{g}$ and angles and $\theta$.

$$
\begin{aligned}
& z_{1}=z_{g}+a \theta+b \phi, \quad z_{2}=z_{g}-a \theta+b \phi, \\
& z_{3}=z_{g}-a \theta-b \phi, \quad z_{4}=z_{g}+a \theta-b \phi .
\end{aligned}
$$

Kinetic energy $T$, elastic energy $V_{i}$ associated with the $i$-th spring of the secondary suspension, and $V_{t j}$ of the $j$-th torsion bar ( $k_{t j}$ is the roll bar stiffness) are described by relations (10) to (12):

$$
\begin{aligned}
& T=\frac{1}{2}\left(m \dot{z}_{g}^{2}+I_{x x} \dot{\phi}^{2}+I_{y y} \dot{\theta}^{2}\right), \\
& V_{i}=\frac{1}{2} k\left(z_{i}-w_{i}\right)^{2}, \quad i=1,2,3,4, \\
& V_{t j}=\frac{1}{2} k_{t}\left(\phi-\frac{w_{j}-w_{j+2}}{2 b}\right)^{2}, \quad j=1,2 .
\end{aligned}
$$

Following the D'Alembert-Lagrange approach and taking into account the forces $u_{i}(i=1,2,3,4)$ exerted by MR actuators, it is possible to obtain the equation system (13) which describes the system's dynamic behavior:

$$
\left.\begin{array}{l}
m \ddot{z}_{g}+4 k z_{g}-k\left(w_{1}+w_{2}+w_{3}+w_{4}\right)=F_{g}, \\
I_{x x} \ddot{\phi}+4 k b^{2} \phi-\left(k b+\frac{k_{t}}{2 b}\right)\left(w_{1}+w_{2}-w_{3}-w_{4}\right)+ \\
k_{t} 2 \phi=M_{x}, \\
I_{y y} \ddot{\theta}+4 k a^{2} \theta-k a\left(w_{1}-w_{2}-w_{3}+w_{4}\right)=M_{y},
\end{array}\right\}
$$

where $F_{g}, M_{x}$, and $M_{y}$ are the equivalent Lagrangian contributions of the non-conservative forces as the $u_{i}$ forces exerted by actuators.

\subsection{Dynamic compensation}

The control strategy aims at compensating the dynamics of the system, namely the control law leads toward 0 the value of the acceleration vector $\ddot{\boldsymbol{x}}=\left(\ddot{z}_{g}, \ddot{\boldsymbol{\phi}}, \ddot{\theta}\right)^{\mathrm{T}}$; as a consequence the control output vector $\boldsymbol{u}=\left(u_{1}, u_{2}, u_{3}, u_{4}\right)^{\mathrm{T}}$ is calculated in order to be exactly equal to the first member of Eq. (13). The linear relation between vector $\boldsymbol{F}_{\mathrm{dc}}=$ $\left(F_{g}, M_{x}, M_{y}\right)$ and the control $\boldsymbol{u}$ is described by (14):

$$
\boldsymbol{F}_{\mathrm{dc}}=\boldsymbol{H u}, \quad \boldsymbol{H}=\left[\begin{array}{cccc}
1 & 1 & 1 & 1 \\
b & b & -b & -b \\
a & -a & -a & a
\end{array}\right] .
$$

Hence, by measuring the accelerations of each actuator on the ends through a double time integration, the positions $z_{i}$ are obtained and, by inverting Eq. (9), the values of the Lagrangian coordinates $\boldsymbol{x}=\left(z_{g}, \phi, \theta\right)^{\mathrm{T}}$ as well as those of their derivatives can be calculated. The measurement of the vectors of disturbances $\boldsymbol{w}=$ $\left(w_{1}, w_{2}, w_{3}, w_{4}\right)^{\mathrm{T}}$ and the first member of Eq. (13) allows the computation of the resulting force and torque values that are required to compensate the system dynamics. To sum up, the control problem consists in finding a vector $\boldsymbol{u}$ that solves Eq. (14): as $\boldsymbol{H}$ is a full-rank matrix, to solve Eq. (14) implies minimizing the difference (15):

$$
\min _{\boldsymbol{u}}\left\|\boldsymbol{F}_{\mathrm{dc}}-\boldsymbol{H} \boldsymbol{u}\right\|^{2}
$$

with $\boldsymbol{u}$ being subject to inequality constraints. The gradient projection method (GPM) [13-14] has been applied to find the solution of Eq. (15). This iterative procedure allows the minimization of a generic function $f(\boldsymbol{u})$, where $\boldsymbol{u}$ is subject to linear equality and inequality constraints of the following form:

$$
\boldsymbol{M u} \leq \boldsymbol{v}_{*} .
$$

In (16), $\boldsymbol{v}_{*}$ is the vector to which is constrained the product $\boldsymbol{M u}$ where $\boldsymbol{M}$ is a weighting matrix; $\boldsymbol{M}$ and $\boldsymbol{v}_{*}$ do not depend on $\boldsymbol{u}$. A particular case is when $f$ is a quadratic form, such as in (15). Here $\boldsymbol{u}$ is subject to inequality constraints as each viscous actuator can only exert a force opposite to the velocity of "deformation" along the vertical axis, i.e. a force that is subject to the inequality constraints (17):

$$
u_{i}(t)=c_{i}\left(\dot{w}_{i}-\dot{z}_{i}\right), 0 \leq c_{0} \leq c_{i} \leq c_{\max }, i=1,2,3,4,(17
$$

where $c_{i}$ is the equivalent damping value imposed on the $i$-th MR actuator. Thus, in the present case, the matrix $\boldsymbol{M}$ in the first member of Eq. (16) is expressed as

$$
\boldsymbol{M}=\left[\begin{array}{cccccccc}
1 & 0 & 0 & 0 & -1 & 0 & 0 & 0 \\
0 & 1 & 0 & 0 & 0 & -1 & 0 & 0 \\
0 & 0 & 1 & 0 & 0 & 0 & -1 & 0 \\
0 & 0 & 0 & 1 & 0 & 0 & 0 & -1
\end{array}\right]^{\mathrm{T}}
$$

The constraint vector $\boldsymbol{v}_{*}$ is not constant, being its 8 entries evaluated as (19) and (20), which correspond to the graph of Fig. 6 :

$$
\begin{aligned}
& v_{i}=\left\{\begin{array}{l}
c_{\max } \text { if }\left(\dot{w}_{i}-\dot{z}_{i}\right)>0, \\
c_{\max } \text { if }\left(\dot{w}_{i}-\dot{z}_{i}\right) \leq 0,
\end{array} i=1,2,3,4 ;\right. \\
& v_{i}=\left\{\begin{array}{l}
-c_{\max } \text { if }\left(\dot{w}_{i}-\dot{z}_{i}\right)<0, \\
c_{\max } \text { if }\left(\dot{w}_{i}-\dot{z}_{i}\right) \geq 0,
\end{array} \quad i=5,6,7,8 .\right.
\end{aligned}
$$




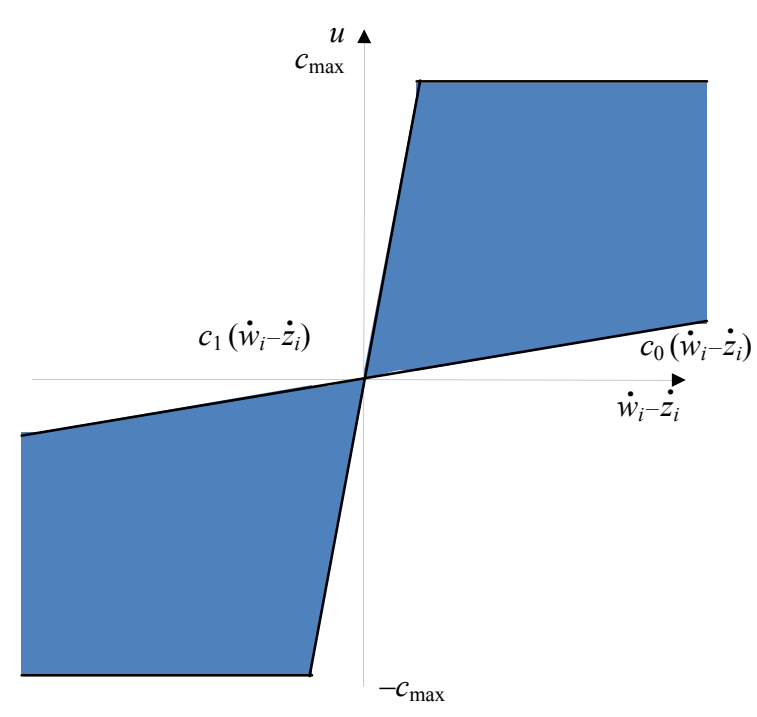

Fig. 6 Allowed values for the control variable $u_{i}$ as a function of the difference with constraints (19) and (20) (dashed area), and with the modified constraints

In conclusion, the GPM takes the vectors $\boldsymbol{w}$ and $\boldsymbol{z}=\left(z_{1}, z_{2}, z, z_{4}\right)^{\mathrm{T}}$ as inputs and finds the control vector $\boldsymbol{u}$ complying to the constraints and solving Eq. (15).

The command $c_{i}$ of each active actuator is obtained from the corresponding control force $u_{i}$ by dividing by $\left(\dot{w}_{i}-\dot{z}_{i}\right)$. Formulation of the constraints described by Eq. (20) suffers from numerical point of view around the null value of $\left(\dot{w}_{i}-\dot{z}_{i}\right)$ causing chattering troubles of the regulator. To address this, the upper bound of $u_{i}$ has been made continuous like the lower bound by expressing it as a straight line with a slope $c_{1}$ far higher than $c_{0}$ as shown in Fig. 6: the corresponding implementation is described by Eqs. (21) and (22).

$$
\begin{aligned}
& v_{i}= \begin{cases}c_{1}\left(\dot{w}_{i}-\dot{z}_{i}\right) & \text { if } 0<\left(\dot{w}_{i}-\dot{z}_{i}\right)<\frac{c_{\max }}{c_{1}}, \\
c_{\max } & \text { if }\left(\dot{w}_{i}-\dot{z}_{i}\right) \geq \frac{c_{\max }}{c_{1}}, \quad i=1,2,3,4, \\
c_{0}\left(\dot{w}_{i}-\dot{z}_{i}\right) & \text { if }\left(\dot{w}_{i}-\dot{z}_{i}\right) \leq 0,\end{cases} \\
& v_{i}=\left\{\begin{array}{l}
c_{1}\left(\dot{w}_{i}-\dot{z}_{i}\right) \text { if }-\frac{c_{\max }}{c_{1}}<\left(\dot{w}_{i}-\dot{z}_{i}\right)<0, \\
-c_{\max } \quad \text { if }\left(\dot{w}_{i}-\dot{z}_{i}\right) \leq-\frac{c_{\max }}{c_{1}}, \quad i=5,6,7,8 .(22) \\
c_{0}\left(\dot{w}_{i}-\dot{z}_{i}\right) \text { if }\left(\dot{w}_{i}-\dot{z}_{i}\right) \geq 0,
\end{array}\right.
\end{aligned}
$$

Finally, in order to assure a longer life and a high reliability of the actuators, the GPM can be induced to research values of the control forces that are quite far from the allowed upper bound. Therefore, a regularization term can be added to modify the function minimized by the GPM algorithm (23):

$$
\min _{\boldsymbol{u}}\left[\left\|\boldsymbol{F}_{\mathrm{dc}}-\boldsymbol{H} \boldsymbol{u}\right\|^{2}+\|\boldsymbol{\Lambda} \boldsymbol{u}\|^{2}\right] .
$$

The square matrix $\boldsymbol{\Lambda}$ is diagonal. The diagonal components of $\boldsymbol{\Lambda}, \lambda_{i i}$, should be chosen according to different criteria: in this work the value of $\lambda_{i i}$ are inversely proportional to the corresponding values of $\left(\dot{w}_{i}-\dot{z}_{i}\right)$. The purpose of the adopted criteria is to prevent the application of excessive values of $c_{i}$ and consequently of $u_{i}$.

\subsection{Dynamic compensation with acceleration feedback}

The approach of the dynamic compensation assumes that the plant estimator describes the behavior of the whole system accurately. Since such model is simplified with respect to the full non-linear multi-body behavior of the vehicle, some performance degradation due to unmodeled dynamics can arise and have to be addressed.

As frequently done on sliding mode regulators [15-16] a feedback term evaluated through a PI (proportional integrator) regulator is introduced. A null acceleration vector of the three Lagrangian coordinates is assumed to be the optimal performance, and the following feedback force $\boldsymbol{F}_{\mathrm{re}}$ aimed at the minimization of the error vector $\boldsymbol{e}=\ddot{\boldsymbol{x}}=\left(\ddot{z}_{g}, \ddot{\phi}, \ddot{\theta}\right)^{\mathrm{T}}$ is applied:

$$
\boldsymbol{F}_{\mathrm{re}}=\boldsymbol{K}_{p}^{\mathrm{T}} \boldsymbol{e}+\boldsymbol{K}_{i}^{\mathrm{T}} \int \boldsymbol{e}=\left[\begin{array}{c}
k_{p z} \ddot{z}_{g}+k_{i z} \dot{z}_{g} \\
k_{p \phi} \ddot{\phi}+k_{i \phi} \dot{\phi} \\
k_{p \theta} \ddot{\theta}+k_{i \theta} \dot{\theta}
\end{array}\right] .
$$

As depicted in the simplified scheme of Fig. 7, the desired vector $\boldsymbol{F}_{\text {tot }}$ is determined to be a sum of the term evaluated through (14) for dynamic compensation and the retro-action calculated in (24), as follows:

$$
\boldsymbol{F}_{\text {tot }}=\boldsymbol{F}_{\mathrm{dc}}+\boldsymbol{F}_{\mathrm{re}} \text {. }
$$

The GPM is exploited in order to find a solution $\boldsymbol{u}$ minimizing (26):

$$
\min _{\boldsymbol{u}}\left[\left\|\boldsymbol{F}_{\text {tot }}-\boldsymbol{H u}\right\|^{2}+\|\boldsymbol{\Lambda} \boldsymbol{u}\|^{2}\right] .
$$

Eq. (26) is solved by imposing inequality constraints:

$$
\begin{aligned}
\boldsymbol{M} & \leq \boldsymbol{v}, \\
\boldsymbol{M} & =\left[\begin{array}{ccccccccccc}
1 & 0 & 0 & 0 & -1 & 0 & 0 & 0 & 1 & b & a \\
0 & 1 & 0 & 0 & 0 & -1 & 0 & 0 & 1 & b & -a \\
0 & 0 & 1 & 0 & 0 & 0 & -1 & 0 & 1 & -b & -a \\
0 & 0 & 0 & 1 & 0 & 0 & 0 & -1 & 1 & -b & a
\end{array}\right] .
\end{aligned}
$$

Vector of constraints, $\boldsymbol{v}$, defined by (28) is similar to that defined by Eqs. (21) and (22) except for the last three elements which have to be introduced since the 


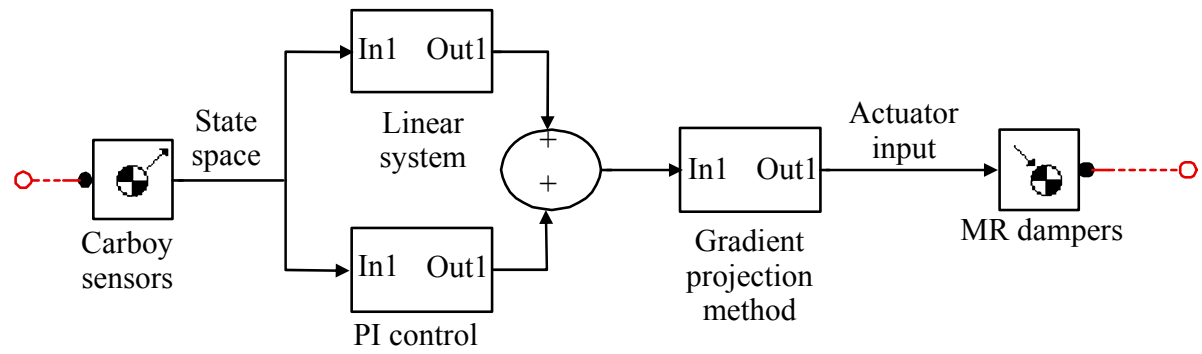

Fig. 7 Control system scheme

size of $\boldsymbol{M}$ is different with respect to the dynamic compensation regulator introduced in Section 4.3:

$$
\boldsymbol{v}=\left[\begin{array}{cc}
i=1,2,3,4 & \text { see Eq. (21) } \\
i=5,6,7,8 & \text { see Eq. (22) } \\
i=9 & \boldsymbol{F}_{g} \\
i=10 & \boldsymbol{M}_{x} \\
i=11 & \boldsymbol{M}_{y}
\end{array}\right] .
$$

\section{Numerical results}

Results obtained with different control techniques are compared with a reference performance, calculated with the same multi-body model of the vehicle assuming a standard passive suspension system. Tests are repeated on the same track with irregularity pattern at different speeds. In Table 1, the same results for index $I_{z}$ defined in (4) are shown: tests are repeated considering different control configurations and traveling speeds, and the in$\operatorname{dex} I_{z}$ is considered a good performance index of the vertical comfort. Generally, all the proposed control techniques improve the comfort of the railway vehicle; however, the dynamic compensation-based approach is negatively affected by the robustness of the plant estimator, while the modified version with an added feedback term shows a much better performance. Noticeably, the performance improvement is higher for the fastest test run, since the imposed irregularities cause higher

Table 1 Performances in terms of $I_{z}$

\begin{tabular}{ccccc}
\hline $\begin{array}{c}\text { Speed } \\
(\mathrm{km} / \mathrm{h})\end{array}$ & Passive & Skyhook & $\begin{array}{c}\text { Dynamic } \\
\text { compensation }\end{array}$ & $\begin{array}{c}\text { Dynamic } \\
\text { compensation } \\
\text { with feedback }\end{array}$ \\
\hline \multirow{2}{*}{140} & 0.136 & 0.122 & 0.12 & 0.079 \\
& $(100 \%)$ & $(89.7 \%)$ & $(88.7 \%)$ & $(58.1 \%)$ \\
200 & 0.204 & 0.187 & 0.190 & 0.104 \\
& $(100 \%)$ & $(91.7 \%)$ & $(93.2 \%)$ & $(50.8 \%)$ \\
250 & 0.276 & 0.230 & 0.221 & 139 \\
& $(100 \%)$ & $(83.4 \%)$ & $(80.2 \%)$ & $(50.2 \%)$ \\
\hline
\end{tabular}

Performances have also been compared in terms of spectral composition of car-body accelerations. For instance, Fig. 8 shows the spectral composition of the vertical accelerations measured on the central-left sensor in Fig. 3. Extending the analysis to a frequency range up to $30 \mathrm{~Hz}$ shows that the spectral component of the measured accelerations still remain below the corresponding performance of the reference passive system. This is a reliable index of robustness and reliability of the control, as there is no-noise regeneration over a wide range of frequencies. This feature is quite useful in terms of system stability, as it involves a lower sensitivity to undamped mechanical modes of the system which may be excited by the action of actuators. Smoothed behavior is also ensured by the capability of the GPM algorithm to find sub optimal actuator set which corresponds to the limited forces as shown in Fig. 9.

On the other hand, skyhook control is much simpler to implement and exhibits a very robust behavior against un-modeled dynamics and even against a failure of one of the actuators as shown in Fig. 10, where the failure is reproduced by cutting off the corresponding command signal. The observed performances are degraded; thus exhibiting a higher rolling motion of carbody. In particular, the unbalanced application of forces due to the

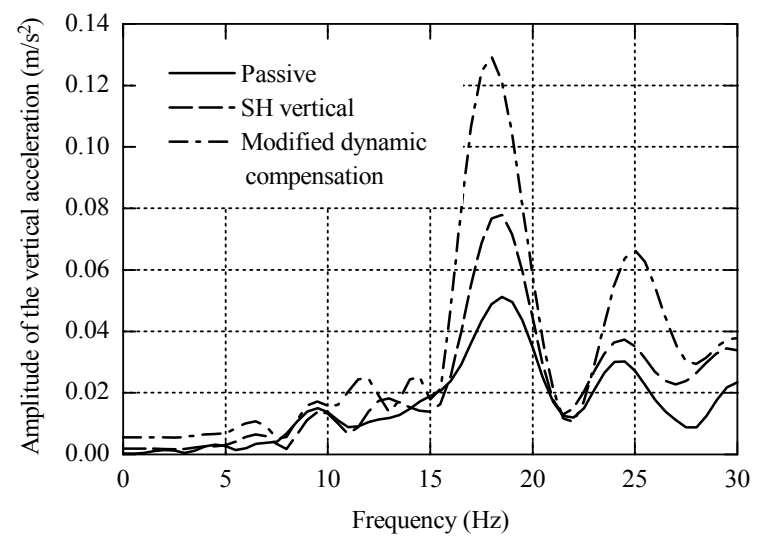

Fig. 8 Spectral composition of vertical acceleration measured on central-left sensor (according to the layout in Fig. 3) 
unavailability of a damper (simulated fault) causes a cross/mutual excitation of roll and pitch modes of the secondary stage suspensions; the behavior, however, is still smooth and the performances are better than in the passive reference simulation.

Finally, the calculated performance indices defined by (4) considering different control configurations and traveling speeds are shown in Tables 2 to 5. From the

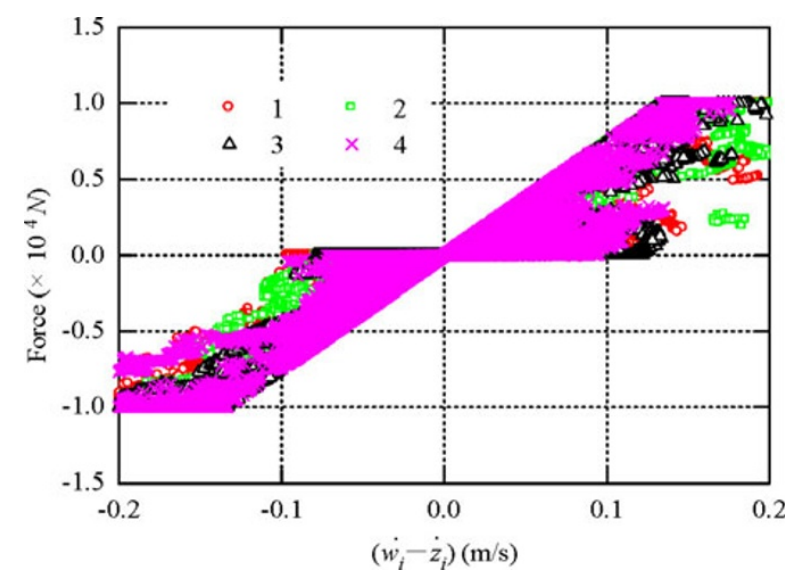

Fig. 9 Actuator forces calculated considering a simulated traveling speed of $250 \mathrm{~km} / \mathrm{h}$ and a control algorithm corresponding to the dynamic compensation with acceleration feedback described in Section 4.4 evaluation of simulation results, it clearly emerges that the proposed control strategies involve comfort benefit in all the three sensor locations, damping all the three comfort-related vibrations of the carbody, such as vertical translation, roll, and pitch rotations. Lateral and longitudinal motions (indices $I_{y}$ and $I_{x}$ ) are less affected by different control strategies since only vertical dampers of the secondary suspension stage are actuated.

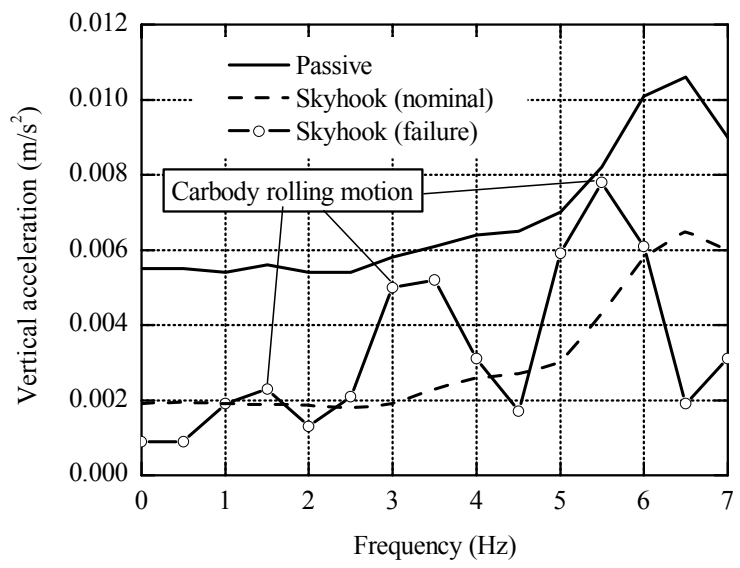

Fig. 10 Spectral composition of vertical acceleration measured on central-left sensor (according to the layout in Fig. 3) considering different working conditions

Table 2 Performance indices for passive reference configuration

\begin{tabular}{ccccccc}
\hline Speed $(\mathrm{km} / \mathrm{h})$ & $I_{1}$ & $I_{2}$ & $I_{3}$ & $I_{x}$ & $I_{y}$ & $I_{z}$ \\
\hline 140 & 0.177 & 0.105 & 0.221 & 0.0144 & 0.108 & 0.136 \\
200 & 0.281 & 0.171 & 0.314 & 0.0229 & 0.164 & 0.204 \\
250 & 0.296 & 0.251 & 0.385 & 0.0262 & 0.150 & 0.276 \\
\hline
\end{tabular}

Table 3 Performance indices calculated for skyhook control

\begin{tabular}{ccccccc}
\hline Speed $(\mathrm{km} / \mathrm{h})$ & $I_{1}$ & $I_{2}$ & $I_{3}$ & $I_{x}$ & $I_{y}$ & $I_{z}$ \\
\hline 140 & 0.167 & 0.102 & 0.206 & 0.0139 & 0.108 & 0.122 \\
200 & 0.260 & 0.174 & 0.290 & 0.0224 & 0.158 & 0.187 \\
250 & 0.256 & 0.221 & 0.326 & 0.0256 & 0.141 & 0.230 \\
\hline
\end{tabular}

Table 4 Performance indices calculated for dynamic compensation

\begin{tabular}{ccccccc}
\hline Speed $(\mathrm{km} / \mathrm{h})$ & $I_{1}$ & $I_{2}$ & $I_{3}$ & $I_{x}$ & $I_{y}$ & $I_{z}$ \\
\hline 140 & 0.170 & 0.098 & 0.197 & 0.0139 & 0.105 & 0.121 \\
200 & 0.260 & 0.179 & 0.290 & 0.0222 & 0.156 & 0.190 \\
250 & 0.250 & 0.214 & 0.325 & 0.0260 & 0.142 & 0.222 \\
\hline
\end{tabular}


Table 5 Performance indices for modified dynamic compensation with acceleration feedback added

\begin{tabular}{ccccccc}
\hline Speed $(\mathrm{km} / \mathrm{h})$ & $I_{1}$ & $I_{2}$ & $I_{3}$ & $I_{x}$ & $I_{y}$ & $I_{z}$ \\
\hline 140 & 0.113 & 0.0745 & 0.143 & 0.0127 & 0.0811 & 0.079 \\
200 & 0.134 & 0.0960 & 0.151 & 0.0170 & 0.0750 & 0.104 \\
250 & 0.179 & 0.1410 & 0.199 & 0.0230 & 0.1040 & 0.139 \\
\hline
\end{tabular}

\section{Conclusions}

According to the results of various simulations, the application of MR dampers to the secondary stage of railway suspension stage and more generally, to the whole suspension system is feasible. The choice and calibration of the control system involve both performance and robustness considerations:

- Despite the poor performance exhibited in simulation, the simple skyhook control is simple and quite insensitive to drastic failures or disturbances due to unmodeled dynamics. Consequently, the performance of this kind of controller may be quite stable even in real operating conditions. In addition, the simple structure of the regulator ensures fast and easy tuning.

- More complex algorithms that coordinate the action of the four actuators as the MIMO regulator provide the best results in simulation. The robustness of the regulator is mainly affected by the capacity of feedback regulator (24) in rejecting errors and disturbances that are not compensated for by the simple estimator model described in Fig. 5. As a consequence, the tuning and robustness have to be carefully verified in a real time application.

Current research in this area focuses on the development of more sophisticated models (considering vehicle car-body flexibility and more sophisticated models of actuators and sensors). Finally, simulation results will be validated by experimental activities performed in Mechatronics and Dynamic Laboratories of University of Florence.

\section{References}

[1] J. An, D.S. Kwon, Modeling of a magnetorheological actuator including magnetic hysteresis, Journal of Intelligent Material Systems and Structures, 2003, 14(9): 541-550.

[2] Lord Materials Division, Designing with MR fluids, rev. 12/99, Engineering Note, Lord Corporation, Thomas
Lord Research Center, Cary, NC, November 1999.

[3] S. Sassi, K. Cherif, L. Mezghani, et al., An innovative magneto-rheological damper for automotive suspension: from design to experimental characterization, Smart $M a$ terials and Structures, 2005, 14(4): 811.

[4] A.H. Lam, W.H. Liao, Semi-active control of automotive suspension systems with magneto-rheological dampers, International Journal of Vehicle Design, 2003, 33(1-3): 50-75.

[5] C.J. Poynor, Innovative Designs for Magneto-Rheological Dampers [Dissertation], Blacksburg, VA: Advanced Vehicle Dynamics Lab, Virginia Polytechnic Institute and State University, 2001.

[6] Lord Corporation-Material Division Engineering, Tech. Note Designing with MR Fluids http://www.lord. com/products-and-solutions/magneto-rheological-(mr)/ military-suspensions.xml, 2010-09-12.

[7] E. Meli, M. Malvezzi, S. Papini, et al., A railway vehicle multibody model for real-time applications, Vehicle System Dynamics, 2008, 46(12): 1083-1105.

[8] L. Pugi, A. Rindi, F. Bartolini, et al., Simulation of a semi-active suspension system for a high speed train, In: Proceedings of IAVSD 2009, Stockholm, Aug. 17-21, 2009.

[9] H. Li, R.M. Goodall, Linear and non-linear skyhook damping control laws for active railway vehicle suspensions, Control Eng. Practice, 1991, 7(7): 843-850.

[10] J.J. Kalker, Wheel-rail rolling contact theory, Wear, 1991, 144: 243-261.

[11] E.C. Bingham, Fluidity and Plasticity, New York: McGraw-Hill, 1922.

[12] Draft prEN 12299 Railway Applications-Ride Comfort for Passengers-Measurement and Evaluation, July 2006 Version.

[13] D.G. Luemberger, Linear and Nonlinear Programming, Addison-Wesley Publishing Company, Reading, Massachusetts, 1984.

[14] B. Allotta, V. Colla, G. Bioli, Kinematic control of robots with joint constraints, Journal of Dynamic Systems, Measurement, and Control, 1999, 121(3): 433-442.

[15] V.I. Utkin, Sliding mode control design principles and applications to electric drives, IEEE Transactions on Industrial Electronics, 1993, 40(1): 23-36.

[16] K. Khalil, Nonlinear Systems, 3rd ed., Upper Saddle River, NJ: Prentice Hall, 2002.

(Editor: Junsi LAN) 